\title{
Modelagem matemática da produtividade e qualidade industrial de grãos de aveia pelo uso do nitrogênio em distintos sistemas de cultivo
}

\author{
Rubia Diana Mantai ${ }^{1}$ \\ Osmar Bruneslau Scremin ${ }^{2}$ \\ Anderson Marolli ${ }^{3}$ \\ Ana Paula Brezolin ${ }^{4}$ \\ Ângela Teresinha Woschinski de Mammann ${ }^{5}$ \\ Ari Higino Scremin ${ }^{6}$ \\ Departamento de Ciências Exatas e Engenharias, UNIJUI, Ijuí, RS \\ José Antonio Gonzalez da Silva ${ }^{7}$ \\ Departamento de Ciências Agrárias, UNIJUI, Ijuí, RS
}

Resumo. A elevada produtividade e qualidade de grãos de aveia é dependente do fornecimento do nitrogênio. Propôs com este estudo determinar o modelo matemático do aproveitamento de nitrogênio em cultivares de aveia na elaboração da produtividade e qualidade industrial de grãos pela máxima eficiência técnica e econômica de uso do nutriente em distintos sistemas de cultivo. O experimento foi delineado em blocos ao acaso com quatro repetições, seguindo um modelo fatorial simples $4 \times 2$, nas fontes de varição doses de $\mathrm{N}$-fertilizante (fonte ureia) nos níveis $0,60,120$ e $180 \mathrm{~kg} \mathrm{~N}^{-1}$ e cultivares de aveia com genótipos URS Taura e URS Tarimba, nos sistemas de sucessão milho/aveia e soja/aveia. Foi realizado teste de médias, e modelos de regressões polinomiais na estimativa da máxima eficiência técnica e econômica de uso do nitrogênio. A cultivar URS Taura mostra-se superior em relação a URS Tarimba quanto à produtividade de grãos e massa do hectolitro, porém, inferior no índice de descasque. No sistema soja/aveia, a dose de $\mathrm{N}$-fertilizante que preconiza a máxima eficiência técnica e econômica de produtividade de grãos é de 98 e 84 $\mathrm{kg}$ de $\mathrm{N} \mathrm{ha}^{-1}$, respectivamente, e produtividade industrial, na máxima eficiência técnica e econômica de 106 e $83 \mathrm{~kg} \mathrm{ha}^{-1}$, respectivamente. No sistema milho/aveia os valores das doses de nitrogênio para a máxima eficiência técnica e econômica foram muito similares devido as condições ambientais satisfatórias para a cultura.

Palavras-chave. Avena sativa, Eficiência técnica, Eficiência econômica, Regressão.

\footnotetext{
${ }^{1}$ rdmantai@yahoo.com.br

2osmarscremin@hotmail.com

${ }^{3}$ marollia@yahoo.com.br

${ }^{4}$ anabrezolin@hotmail.com

${ }^{5}$ angelademamann@hotmail.com

6 ahscremin@hotmail.com

7 jagsfaem@yahoo.com.br
} 


\section{Introdução}

A aveia branca é um cereal de inverno com múltiplos propósitos, como cobertura e proteção do solo e usos na alimentação animal e humana [2]. Seus grãos possuem um alto valor nutritivo, rico em fibras e proteínas, qualificando a espécie e evidenciando o interesse industrial [1]. Esta espécie é altamente dependente de nitrogênio, nutriente fundamental para seu crescimento e desenvolvimento [4].

$\mathrm{O}$ emprego adequado do $\mathrm{N}$-fertilizante para a cultura pode promover elevada produtividade e qualidade, mas também pode ocorrer prejuízos, pelo excesso do nutriente, reduzindo a produtividade e/ou causando o acamamento da plantas [2]. Portanto, além do uso adequado do $\mathrm{N}$-fertilizante, é fundamental a utilização de cultivares com capacidade de absorção e uso para alta expressão de caracteres ligados a produtividade de grãos e de indútria.

As condições climáticas e técnicas de cultivo também atuam sobre a eficiência de uso e aproveitamento do nitrogênio $[3,6]$, pois, o N-residual modifica a dinâmica de uso do $\mathrm{N}$-fertilizante pela planta, através do tipo de precedente de alta ou reduzida decomposição da palha [7]. Portanto, a necessidade constante de análise sobre novos genótipos à quantificação da máxima eficiência técnica e econômica de uso do nitrogênio [3].

O objetivo do estudo é determinar o modelo matemático do aproveitamento de nitrogênio em cultivares de aveia na elaboração da produtividade e qualidade industrial de grãos pela máxima eficiência técnica e econômica de uso do nutriente em distintos sistemas de cultivo.

\section{Metodologia}

O trabalho foi desenvolvido a campo no ano agrícola de 2015 no Instituto Regional de Desenvolvimento Rural (IRDeR) do Departamento de Estudos Agrários (DEAg) da UNIJUÍ. A semeadura foi realizada na primeira semana de junho com semeadoraadubadora para composição da parcela constituída de 5 linhas de $5 \mathrm{~m}$ de comprimento e espaçamento entre linhas de $0,20 \mathrm{~m}$, formando a unidade experimental de $5 \mathrm{~m}^{2}$.

$\mathrm{O}$ experimento foi delineado em blocos ao acaso com quatro repetições, seguindo um modelo fatorial simples $4 x 2$, nas fontes de variação doses de $\mathrm{N}$-fertilizante (fonte ureia) nos níveis $0,60,120$ e $180 \mathrm{~kg} \mathrm{~N}^{-1}$ e cultivares de aveia com genótipos elite URS Taura e URS Tarimba, nos sistemas de sucessão milho/aveia e soja/aveia, totalizando 32 unidades experimentais por sistema de cultivo.

Os dados foram submetidos à análise de variância (ANOVA), teste de comparação de médias pelo modelo de Scott e Knott, conforme expressão,

$$
\lambda=\frac{\pi}{2(\pi-2)} \cdot \frac{\beta_{0}}{\hat{\sigma_{0}^{2}}}
$$

em que, $\beta_{0}$ é o valor máximo das somas de quadrados entre grupos de médias de tratamentos, $\sigma_{0}^{2}$ é a estimativa de máxima verossimilhança de $\sigma^{2}$, dada por, 


$$
\sigma_{0}^{2}=\frac{\sum_{i=1}^{p}\left(\bar{y}_{i}-\bar{y}\right)^{2}+v s^{2}\left(\bar{y}_{i}\right)}{(p+v)}
$$

em que, $\bar{y}_{i}$ é a média do tratamento $i, \bar{y}$ é a média de todos os dados, $s^{2}\left(\bar{y}_{i}\right)$ é a estimativa da variância da média do tratamento $i, n$ é o número de repetições, $p$ é o número de tratamentos, e $v$ é o número de graus de liberdade do resíduo da ANOVA.

Foram realizadas regressões para ajuste do grau de polinômio visando estabelecer a máxima eficiência técnica e econômica das variáveis de interesse pela equação,

$$
y=a \pm b x \pm c x^{2}
$$

em que $y$ é a variável resposta, $x$ é a dose de N-fertilizante, e $a, b$, e $c$ são coeficientes do modelo. A partir da equação 3 foi empregado o modelo matemático da estimativa da máxima eficiência técnica, dado por,

$$
x=\frac{-b}{2 c}
$$

e o modelo da máxima eficiência econômica, dado por,

$$
x=\frac{\left(\frac{t}{w}\right) \cdot(-b)}{2 c}
$$

em que $t$ é o valor do insumo (ureia) e $w$ o valor do produto (aveia branca), sendo que neste período, o quilograma de ureia correspondeu ao custo de $\mathrm{R} \$ 1,10$ e o valor pago ao produtor pelo $\mathrm{kg}$ de aveia branca em $\mathrm{R} \$ 0,90$.

\section{Resultados e Discussão}

Na Tabela 1, na análise do sistema soja/aveia, o efeito das doses de N-fertilizante foram significativos em alterar a produtividade de grãos e de indústria. Nos genótipos testados, houve significância nos caracteres massa do hectolitro $(\mathrm{MH})$, índice de descasque (ID) e produtividade de grãos (PG). No sistema milho/aveia, foi observado que os efeitos de doses foram significativos em alterar a PG, a produtividade industrial (PI) e a $\mathrm{MH}$.

Na comparação de médias entre as cultivares (Tabela 2), no sistema soja/aveia, a cultivar URS Taura mostrou maior expressão na PG e MH, porém, a URS Tarimba foi superior no ID. No sistema milho/aveia não houve alteração da PG e ID, apenas para a $\mathrm{MH}$, onde a cultivar URS Taura foi superior.

Estudos realizados em aveia salientam que a recomendação da cultivar ao produtor é decisiva na produtividade e qualidade industrial de grãos [1]. Desta forma, é fundamental o ajuste da eficiência de uso do nitrogênio sobre as cultivares disponíveis, promovendo produções economicamente satisfatórias e com menor risco de poluição ambiental [3].

Na Tabela 3, no sistema soja/aveia, equações quadráticas foram significativas para a PG e PI. Assim, a MET e MEE na PG foi de 98 e $84 \mathrm{~kg}$ de $\mathrm{N} \mathrm{ha}^{-1}$, numa estimativa de 3439 e $3430 \mathrm{~kg} \mathrm{ha}^{-1}$, respectivamente. Na PI, a dose para a MET foi de $106 \mathrm{~kg}$ de $\mathrm{N} \mathrm{ha}^{-1}$ com uma PG de $1997 \mathrm{~kg} \mathrm{ha}^{-1}$ e MEE de $83 \mathrm{~kg}$ de $\mathrm{N} \mathrm{ha}^{-1}$ com $1982 \mathrm{~kg} \mathrm{ha}{ }^{-1}$. 
Tabela 1: Resumo da análise de variância dos efeitos de doses de nitrogênio no cultivo de diferentes genótipos de aveia branca na expressão dos caracteres de produção e qualidade industrial sobre resíduo de soja e milho.

\begin{tabular}{|c|c|c|c|c|c|c|c|}
\hline \multirow{2}{*}{$\begin{array}{l}\text { Fonte de } \\
\text { Variação }\end{array}$} & \multirow[b]{2}{*}{ GL } & \multicolumn{6}{|c|}{ Quadrado Médio/ Caracteres de produção e qualidade industrial } \\
\hline & & $\begin{array}{c}\mathrm{PG} \\
\left(\mathrm{kg} \mathrm{ha}^{-1}\right)\end{array}$ & $\begin{array}{c}\mathrm{MH} \\
\left(\mathrm{kg} \mathrm{hL}^{-1}\right)\end{array}$ & $\begin{array}{l}\text { MMG } \\
(\mathrm{g})\end{array}$ & $\begin{array}{c}\mathrm{NG}>2 \mathrm{~mm} \\
(\mathrm{n})\end{array}$ & $\begin{array}{c}\text { ID } \\
\left(\mathrm{g} \mathrm{g} \mathrm{g}^{-1}\right)\end{array}$ & $\begin{array}{c}\text { PI } \\
\left(\mathrm{kg} \mathrm{ha}^{-1}\right)\end{array}$ \\
\hline \multicolumn{8}{|c|}{ sistema soja/aveia } \\
\hline Bloco & 3 & 93072 & 0,53 & 0,49 & 33,7 & 0,0001 & 10348 \\
\hline Dose (D) & 3 & $369885^{*}$ & $1,44^{n s}$ & $2,03^{n s}$ & $22,70^{n s}$ & $0,003^{n s}$ & $227450^{*}$ \\
\hline Genótipo (G) & 1 & $405675^{*}$ & $9,03^{*}$ & $0,004^{n s}$ & $10,12^{\text {ns }}$ & $0,132^{*}$ & $32131^{\text {ns }}$ \\
\hline D X G & 3 & $92711^{\text {ns }}$ & $1,94^{n s}$ & $2,01^{n s}$ & $12,20^{n s}$ & $0,005^{n s}$ & $83501^{\text {ns }}$ \\
\hline Erro & 21 & 88302 & 0,95 & 1,54 & 9,42 & 0,002 & 51741 \\
\hline Total & 31 & & & & & & \\
\hline Média Geral & & 3246 & 56 & 33,75 & 75,56 & 0,76 & 1871 \\
\hline CV (\%) & 9,15 & 1,74 & 3,68 & 4,06 & 6,1 & 12,15 & \\
\hline \multicolumn{8}{|c|}{ sistema milho/aveia } \\
\hline Bloco & 3 & 70232 & 1,75 & 0,4 & 4,69 & 0,0007 & 34879 \\
\hline Dose (D) & 3 & $4165767^{*}$ & $17,41^{*}$ & $3,26^{n s}$ & $15,36^{n s}$ & $0,0038^{n s}$ & $1238090^{*}$ \\
\hline Genótipo (G) & 1 & $85594^{\text {ns }}$ & $6,12^{*}$ & $0,55^{n s}$ & $11,28^{n s}$ & $0,0003^{n s}$ & $11666^{n s}$ \\
\hline D X G & 3 & $37197^{n s}$ & $2,2^{\text {ns }}$ & $1,38^{n s}$ & $11,36^{n s}$ & $0,0023^{n s}$ & $33786^{n s}$ \\
\hline Erro & 21 & 28364 & 0,72 & 2,05 & 15,62 & 0,0017 & 18158 \\
\hline Total & 31 & & & & & & \\
\hline Média Geral & & 2856 & 54,87 & 32,67 & 72,4 & 0,72 & 1497 \\
\hline CV (\%) & & 5,89 & 1,55 & 4,38 & 5,45 & 5,69 & 8,99 \\
\hline
\end{tabular}

*=significativo a nível de $5 \%$ de probabilidade de erro pelo teste $\mathrm{F}$; ns= não significativo a nível de $5 \%$ de probabilidade de erro pelo teste $\mathrm{F}$; $\mathrm{CV}=$ Coeficiente de variação; $\mathrm{GL}=$ graus de liberdade; $\mathrm{PG}=\mathrm{Produtividade} \mathrm{de} \mathrm{grãos;}$ $\mathrm{MH}=$ Massa do hectolitro; $\mathrm{MMG}=$ Massa de mil grãos; $\mathrm{NG}>2 \mathrm{~mm}=$ Número de grãos maior que $2 \mathrm{~mm}$; ID= índice de descasque; PI= Produtividade industrial.

Tabela 2: Comparação de médias dos caracteres para a produtividade e qualidade industrial para distintas cultivares e sistemas de plantio da cultura da aveia branca.

\begin{tabular}{lccc}
\hline Cultivares & PG $\left(\mathrm{kg} \mathrm{ha}^{-1}\right)$ & $\mathrm{MH}\left(\mathrm{kg} \mathrm{hL}^{-1}\right)$ & $\mathrm{ID}\left(\mathrm{g} \mathrm{g}^{-1}\right)$ \\
\hline \multirow{4}{*}{ URS Tarimba } & $3133 \mathrm{~b}$ & $56 \mathrm{~b}$ & \\
URS Taura & $3358 \mathrm{a}$ & $57 \mathrm{a}$ & $0,78 \mathrm{a}$ \\
\hline \multicolumn{4}{c}{ sistema milho/aveia } \\
URS Tarimba & - & $54,43 \mathrm{~b}$ & $0,74 \mathrm{~b}$ \\
URS Taura & - & $55,31 \mathrm{a}$ & - \\
\hline
\end{tabular}

Médias seguidas de mesma letra na coluna não diferem entre si na probabilidade de $5 \%$ de erro pelo teste de Scott e Knott. $\mathrm{PG}=$ Produtividade de grãos; $\mathrm{MH}=$ Massa do hectolitro; $\mathrm{ID}=$ índice de descasque.

A diferença de uso do nitrogênio pela eficiência técnica e econômica se mostrou expressiva, de modo que para a PG, a MEE reduziu $14 \mathrm{~kg} \mathrm{ha}^{-1}$ de $\mathrm{N}$ mantendo a produtividade 
Tabela 3: Determinação da equação e grau do polinômio nas variáveis dependentes do rendimento de grãos e qualidade industrial, valores da estimativa da máxima eficiência técnica (MET) e econômica de produção (MEE) pelos efeitos de doses de nitrogênio em aveia.

\begin{tabular}{|c|c|c|c|c|c|c|}
\hline $\begin{array}{l}\text { Equação } \\
y=a \pm b x \pm c x^{2}\end{array}$ & $\mathrm{R}^{2}$ & $\begin{array}{c}\mathrm{P} \\
\left(b_{i x}^{n}\right)\end{array}$ & $\begin{array}{c}\text { MET } \\
\mathrm{N}\left(\mathrm{kg} \mathrm{ha}^{-1}\right)\end{array}$ & $\begin{array}{c}\text { MET } \\
y_{E}\end{array}$ & $\begin{array}{c}\text { MEE } \\
\mathrm{N}\left(\mathrm{Kg} \mathrm{ha}^{-1}\right)\end{array}$ & $\begin{array}{c}\mathrm{MEE} \\
y_{E}\end{array}$ \\
\hline \multicolumn{7}{|c|}{ sistema soja/aveia } \\
\hline$P G=3030+8,3113 x-0,0122 x^{2}$ & 79 & $*$ & 98 & 3439 & 84 & 3430 \\
\hline$P I=1700+5,5756 x-0,0261 x^{2}$ & 87 & $*$ & 106 & 1997 & 83 & 1982 \\
\hline \multicolumn{7}{|c|}{ sistema milho/aveia } \\
\hline$P G=1817+26,7157 x-0,1083 x^{2}$ & 98 & $*$ & 123 & 3464 & 117 & 3460 \\
\hline$M H=53+0,0422 x-0,0001 x^{2}$ & 84 & $\mathrm{~ns}$ & - & - & - & - \\
\hline$P I=931+13,4530 x-0,0511 x^{2}$ & 99 & $*$ & 131 & 1816 & 119 & 1808 \\
\hline
\end{tabular}

de grãos praticamente inalterada (Tabela 3). Do mesmo modo para a PI, com uma redução de aproximadamente $20 \%$ na dose de $\mathrm{N}$-fertilizante. Estes dados, confirmam que a dose da eficiência econômica além de garantir uma boa produção, ainda proporciona menores gastos ao produtor

Na Tabela 3, no sistema milho/aveia, a MH apresentou uma tendência quadrática, mas não significativa, já as demais variáveis obtiveram-se equações quadráticas com parâmetro de grau dois efetivo. Na PG, a dose estimada à MET e MEE foi de 123 e $117 \mathrm{~kg}$ de N $\mathrm{ha}^{-1}$, numa PG estimada em 3464 e $3460 \mathrm{~kg} \mathrm{ha}{ }^{-1}$, respectivamente. Na PI os valores para a MET e MEE foram de $131 \mathrm{e} 119 \mathrm{~kg}$ de $\mathrm{N} \mathrm{ha}^{-1}$, respectivamente, com PI estimado de 1816 e $1808 \mathrm{~kg} \mathrm{ha}^{-1}$, respectivamente. O valor da dose de nitrogênio para a PG e PI obtido pela MET e MEE foi muito similar, devido a um ano agrícola favorável para a aveia (Figura 1), com constantes precipitações ao longo do ciclo de desenvolvimento, permitindo alavancar a absorção do nutriente.

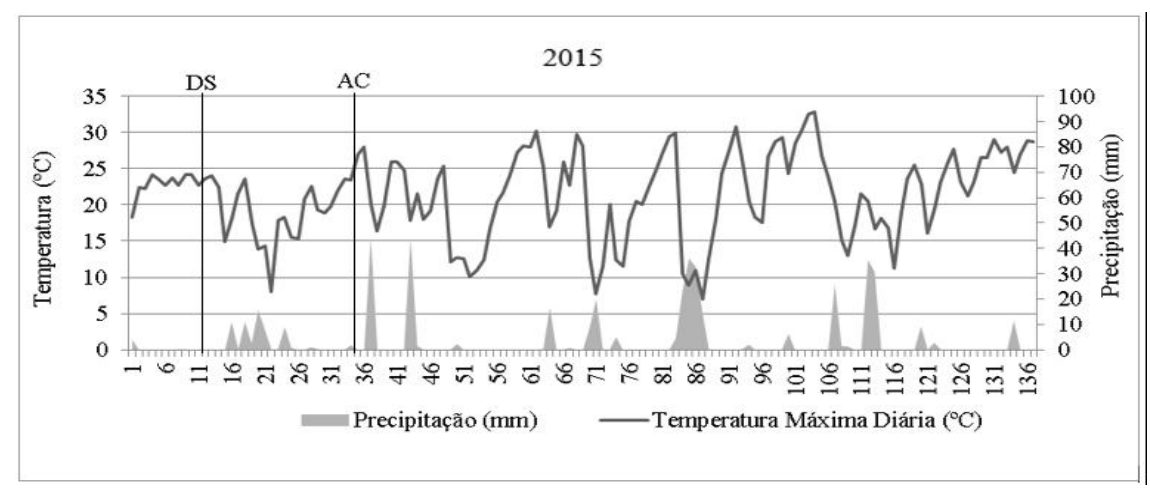

Figura 1. Dados de precipitação pluviométrica e temperatura máxima do ano de 2015;

DS - Data da Semeadura; AC - Adubação em Cobertura. Fonte: Instituto Regional de Desenvolvimento Rural.

O aumento de precipitação pluviométrica proporciona maiores condições para decom- 
posição do resíduo de cobertura sobre o solo, melhorando a liberação de nutrientes para o aproveitamento das plantas em sucessão [5].

\section{Conclusões}

A cultivar URS Taura mostra-se superior em relação a URS Tarimba quanto à produtividade de grãos e massa do hectolitro, porém, inferior no índice de descasque.

No sistema soja/aveia, a dose de N-fertilizante que preconiza a máxima eficiência técnica e econômica de produtividade de grãos é de 98 e $84 \mathrm{~kg}$ de $\mathrm{N} \mathrm{ha}^{-1}$, respectivamente, e produtividade industrial, na eficiência técnica e econômica de 106 e $83 \mathrm{~kg} \mathrm{ha}^{-1}$, respectivamente.

No sistema milho/aveia os valores das doses de nitrogênio para a máxima eficiência técnica e econômica foram muito similares devido as condições ambientais satisfatórias para a cultura.

\section{$5 \quad$ Agradecimentos}

Ao $\mathrm{CNPq}$, CAPES, FAPERGS e à UNIJUI pelo aporte dos recursos destinados ao desenvolvimento deste estudo e pelas bolsas de Iniciação Científica e Tecnológica, de Apoio Técnico, de Pós-graduação e de Produtividade em Pesquisa.

\section{Referências}

[1] M. Crestani, F. I. F. de Carvalho, A. C. de Oliveira, J. A. G. da Silva, L. C. Gutkoski, J. F. Sartori, R. L. Barbieri, D. Baretta. Conteúdo de $\beta$-glucana em cultivares de aveia branca cultivadas em diferentes ambientes, Pesquisa Agropecuária Brasileira, $45: 261-268,2010$.

[2] M. C. Hawerroth, J. A. G. da Silva, C. A. Souza, A. C. de Oliveira, H. de S. Luche, C. M. Zimmer, F. J. Hawerroth, J. Schiavo, J. C. Sponchiado. Redução do acamamento em aveia branca com uso do regulador de crescimento etil trinexapac, Pesquisa Agropecuária Brasileira, 50 : 115 - 125, 2015. DOI: 10.1590/S0100 - 204X2015000200003.

[3] R. D. Mantai, J. A. G. da Silva, A. T. Z. R. Sausen, J. S. P. Costa, S. B. V. Fernandes, C. Ubessi. A eficiência na produção de biomassa e grãos de aveia pelo uso do nitrogênio, Revista Brasileira de Engenharia Agrícola e Ambiental, 19 : 343 - 349, 2015. DOI: 10.1590/1807-1929/agriambi.v19n4p343-349.

[4] L. Sangoi, A. C. Berns, M. L. de Almeida, C. G. Zanin, C. Schweitzer. Características agronômicas de cultivares de trigo em resposta à época da adubação nitrogenada de cobertura, Ciência Rural, 37:1564-1570, 2007. DOI:10.1590/S010384782007000600010 . 
[5] J. A. G. da Silva, M. B. da Motta, C. A. M. Bianchi, M. Crestani, J. Gaviraghi, C. Fontaniva, E. Gewbwe. Alelopatia da canola sobre o desenvolvimento e produtividade da soja. Revista Brasileira de Agrociência, 17:428-437, 2011.

[6] R. Viola, G. Benin, L. C. Cassol, C. Pinnow, M. F. Flores, E. Bornhofen. Adubação verde e nitrogenada na cultura do trigo em plantio direto, Bragantia, 72:20-28, 2013. DOI:10.1590/ S0006-87052013005000013.

[7] A. Wendling, F. L. F. Eltz, M. M. Cubilla, T. J. C. Amado, J. Mielniczuk, T. Lovato. Recomendação de adubação nitrogenada para trigo em sucessão ao milho e soja sob sistema plantio direto no Paraguai, Revista Brasileira de Ciência do Solo, 31:985-994, 2007. DOI:10.1590/S0100-06832007000500015. 